\title{
INFLUÊNCIA DA URBANIZAÇÃO NA QUALIDADE DA ÁGUA DO RIO BRANCO E AFLUENTES NO MUNICÍPIO DE BOA VISTA, RORAIMA, AMAZÔNIA BRASILEIRA
}

\author{
URBANIZATION INFLUENCE ON THE WATER QUALITY OF \\ THE BRANCO RIVER AND TRIBUTARIES IN BOA VISTA, \\ RORAIMA, BRAZILIAN AMAZON
}

Andréa Cristina Sant'Ana ${ }^{1}$, Marcos José Salgado Vital ${ }^{1}$, Henrique Eduardo Bezerra da Silva ${ }^{1}$

${ }^{1}$ Universidade Federal de Roraima - UFRR, Campus do Paricarana, Boa Vista, RR, Brasil. E-mails: andreasantana290607@gmail.com, salvital2@hotmail.com, hebsquim@globo.com.

\begin{abstract}
Como citar: SANT'ANA, A. C.; VITAL, M. J. S.; SILVA, H. E. B. Influência da urbanização na qualidade da água do Rio Branco e afluentes no município de Boa Vista, Roraima, Amazônia brasileira. Revista de Gestão de Água da América Latina, v. 16, e6, 2019.

https://dx.doi.org/10.21168/rega.v16e6.
\end{abstract}

RESUMO: 0 crescente processo de urbanização da cidade de Boa Vista (Roraima) vem interferindo sobremaneira na conformação natural do rio Branco e de seus afluentes. Determinar a influência da urbanização na qualidade da água superficial que abastece esta cidade é imprescindível para realizar uma melhor gestão pública dos recursos hídricos locais. Desta forma, foram estabelecidos 18 pontos de coleta de água distribuídos ao longo do rio Branco e de seus principais afluentes. As coletas tiveram periodicidade mensal (janeiro a junho/2006). Os parâmetros de qualidade de água utilizados como variáveis independentes foram: demanda bioquímica de oxigênio, cloreto, nitrato, sólidos totais dissolvidos, condutividade elétrica, oxigênio dissolvido, alcalinidade, potencial hidrogeniônico, temperatura, turbidez e coliformes termotolerantes. Índice de Qualidade da Água (IQA-RR) e técnicas de análise multivariada (Análise de Componentes Principais - PCA e Análise Hierárquica de Cluster - HCA) indicaram uma separação clara entre os pontos de coleta, formando três grupos: (i) "G1" com bom nível de qualidade, situado fora do ambiente urbano, (ii) "G2" com nível médio de qualidade, próximo ao ambiente urbano e com presença de balneários públicos e (iii) "G3" com nível de qualidade muito ruim, inserido no ambiente urbano. As variáveis responsáveis por esse agrupamento foram demanda bioquímica de oxigênio, condutividade, sólidos totais dissolvidos, nitrato, cloreto e coliformes termotolerantes. Houve a indicação de que a poluição de origem orgânica é a principal responsável pela diminuição da qualidade da água superficial. Informações desta natureza são passos importantes no entendimento do impacto da urbanização sobre os recursos hídricos locais e são base para a construção de políticas públicas que mitiguem os impactos negativos da expansão urbana sem planejamento.

Palavras-chave: Poluição hídrica, Análise de Componentes Principais, Análise Hierárquica de Cluster, Índice de Qualidade da Água

ABSTRACT: The increasing urbanization process of the city of Boa Vista (Roraima State) has been interfering greatly in natural conformation of the Branco river and its tributaries. Determining the influence of urbanization on the quality of surface water that supplies this city is essential to achieve a better public management of local water resources. In this way, 18 points of water collection were established along the Branco river and its main tributaries. Samples were collected monthly (January to June / 2006). Water quality parameters used as independent variables were biochemical oxygen demand, chloride, nitrate, total dissolved solids, electrical conductivity, dissolved oxygen, alkalinity, hydrogenation potential, temperature, turbidity and thermotolerant coliforms. Principal Component Analysis - PCA and Hierarchical Cluster Analysis - HCA indicated a clear separation among the collection points, forming three groups: (i) "G1" with good water quality index located outside the urban environment; (ii) "G2" with a medium quality level, close to the urban environment and public bathhouses; and (iii) "G3" with a very poor quality level, inserted in the urban environment. The variables responsible for grouping were biochemical oxygen demand, conductivity, total dissolved solids, nitrate, chloride and thermotolerant coliforms. Data analysis indicated that

Recebido: 18/11/2018. Revisado: 01/04/2019. Aceito: 18/06/2019. 
pollution of organic origin is primarily responsible for reduction of surface water quality. Informations of this nature are important to understand the impact of urbanization on local water resources and are the basis for construction of public policies that mitigate negative impacts of urban sprawl without planning.

Keywords: Water Pollution, Principal Component Analysis, Hierarchical Cluster Analysis, Water Quality Index

\section{INTRODUÇÃO}

Muitos trabalhos realizados ao longo dos anos, mostram que a degradação dos recursos hídricos está diretamente relacionada à intervenção antrópica, que traz consigo processos que alteram as condições naturais do ambiente aquático, sendo por meio de desmatamento, da expansão da agricultura, da abertura de estradas ou da urbanização (ARAÚJO JUNIOR; TAVARES JUNIOR, 2018; VALLIM; TRAVASSOS, 2019).

Nas últimas décadas, o Brasil vem passando por intenso processo de intervenção antrópica, primeiramente na região Sul e Sudeste, que hoje concentram imensos contingentes habitacionais em grandes centros urbanos, atingindo seu auge na década de 1950, migrando, nas décadas seguintes, para as regiões Nordeste e Centro-Oeste, intensificando-se por derradeiro na região Norte (LUCKMANN, 1989; INSTITUTO BRASILEIRO DE GEOGRAFIA E ESTATÍSTICA, 2010).

0 crescimento demográfico na região Norte, trouxe consigo todos os problemas inerentes a ocupação humana, o que afetou diretamente a qualidade de suas águas. Alguns trabalhos mostram que os índices de poluição nas zonas urbanas da região têm se mostrado significativamente altos, podendo ser comparáveis a níveis encontrados em países subdesenvolvidos e/ou altamente industrializados (BECKER, 1998, 2003; TUNDISI, 2014; ARAÚJO JUNIOR; TAVARES JUNIOR, 2018).

Os problemas acima expostos, mostram à importância de se intensificar estudos ligados a qualidade da água superficial que servem os estados da região Norte, área amazônica rica em recursos hídricos, com o intuito de se gerar informações científicas acerca da influência dos processos de urbanização nesses ambiente aquáticos, proporcionando indicadores necessários para mudanças de rumo nas políticas públicas voltadas à conservação e preservação dos recursos da região (BECKER, 2003; TOZI; MASCARENHAS; PÓLEN, 2018).

0 estado de Roraima, inserido na região Norte, possui riqueza em volume de águas superficiais. 0 principal tributário de sua rede de drenagem é o rio Branco, formado pela confluência dos rios Uraricoera e Tacutu; ele deságua no rio Negro, seu maior afluente (CARVALHO; MORAIS, 2014), e traz algumas peculiaridades em seu processo de urbanização. Roraima teve crescimento populacional explosivo relacionado às migrações ocorridas por conta do garimpo nas décadas de 1970/1980, o que acarretou um processo de ocupação humana acelerado e caótico, principalmente em sua capital Boa Vista, que atualmente possui o maior índice populacional do Estado (a saber: índice populacional de Roraima: 2,01 hab/km2; índice de Boa Vista: 57,4 hab/ $\mathrm{km}^{2}$ ), e mesmo com o processo de ocupação humana diminuindo após o fechamento dos garimpos, seu crescimento populacional ainda é significativo (INSTITUTO BRASILEIRO DE GEOGRAFIA E ESTATÍSTICA, 2010; FALCÃO et al., 2012; ARAUJO JUNIOR; TAVARES JUNIOR, 2018).

Diante dos processos de urbanização instalados, que podem afetar águas superficiais locais, as políticas públicas estaduais voltadas aos recursos hídricos são incipientes e apresentam diversas lacunas. 0 Estado, foi a última unidade da federação brasileira a introduzir a Política Estadual de Recursos Hídricos, com base na Lei 9.433/97 (TOSCANO; SILVA, 2012; TOZI; MASCARENHAS; PÓLEN, 2018). Uma rede de monitoramento da qualidade da água superficial foi implantada, como parceria entre o Governo Estadual e a Universidade Federal de Roraima, mas funcionou por curto período de tempo, o que não a fez cumprir o papel a que se destinava (VITAL, 2004). Também, os estudos inerentes ao tema, desenvolvidos pela comunidade científica local ainda são escassos.

Com base no acima exposto elaboramos o presente estudo, que objetivou a determinação da influência que processos de urbanização exercem na qualidade da água do rio Branco e afluentes, no município de Boa Vista, Roraima. Informações desta natureza são passos importantes no entendimento do impacto que a urbanização exerce sobre os rios e igarapés da Capital, e pode se tornar base para a construção de políticas públicas direcionadas à proteção dos recursos hídricos locais.

\section{METODOLOGIA}

Nosso sítio de estudo foi o município de Boa Vista, capital do estado de Roraima, situado na margem direita do rio Branco e cortado por diversos igarapés, afluentes deste corpo hídrico. Boa Vista registrou um total de 277.799 habitantes pelo senso de 2010, sendo considerado o maior centro 
urbano de Roraima (INSTITUTO BRASILEIRO DE GEOGRAFIA E ESTATÍSTICA, 2010). A Capital possui uma área de aproximadamente $5.687,0 \mathrm{Km}^{2}$, estando situada no setor centro-sul da maior área de savana do extremo norte Amazônico (BARBOSA; CAMPOS, 2011). 0 tipo climático dominante na cidade é o Aw, classificação de Köppen (tropical com fortes chuvas de verão), onde predominam temperaturas médias anuais de aproximadamente $26^{\circ} \mathrm{C}$, com período seco entre outubro-março, e período chuvoso entre abril-setembro, onde são concentrados $\sim 80 \%$ do total precipitado anualmente (ALVARES et al., 2014; SILVA et al., 2015).

Foram utilizadas 18 unidades amostrais (Figura 1), demarcadas previamente pela rede de monitoramento de qualidade de água do município de Boa Vista, de acordo com Vital (2004). Os pontos foram localizados nos ambientes de confluência de afluentes do rio Branco que cortam a Capital, e, nos casos da drenagem na área urbana, em pontos onde os rios e igarapés já tinham sido amostrados anteriormente, mais de uma vez.

Em cada unidade amostral foram coletadas amostras simples de água superficial, com periodicidade mensal, de janeiro a junho de 2006. Todas as coletas foram realizadas no período da manhã, e a metodologia de coleta, transporte, armazenamento e conservação das amostras seguiram o preconizado no Standard Methods for Examination of Water and Wastewater (AMERICAN PUBLIC HEALTH ASSOCIATION, 1999).

Para as análises físico-químicas e microbiológicas foram escolhidos os parâmetros indicadores de poluição hídrica preconizados pela Resolução CONAMA 357/05 (BRASIL, 2005), já utilizados na rotina do Laboratórios de Águas da Universidade Federal de Roraima para rede de monitoramento da qualidade de água do município de Boa Vista, a saber: potencial hidrogeniônico (pH), condutividade elétrica (CE), sólidos totais dissolvidos (STD), temperatura (TEMP), oxigênio dissolvido (OD), turbidez (TURB), alcalinidade (ALC), cloreto ( $\mathrm{Cl}$ ), nitrato $\left(\mathrm{NO}_{3}\right)$, demanda bioquímica de oxigênio a $20^{\circ} \mathrm{C}$ por cinco dias $\left(\mathrm{DBO}_{5,20}\right)$, coliformes termotolerantes (COL TERM).

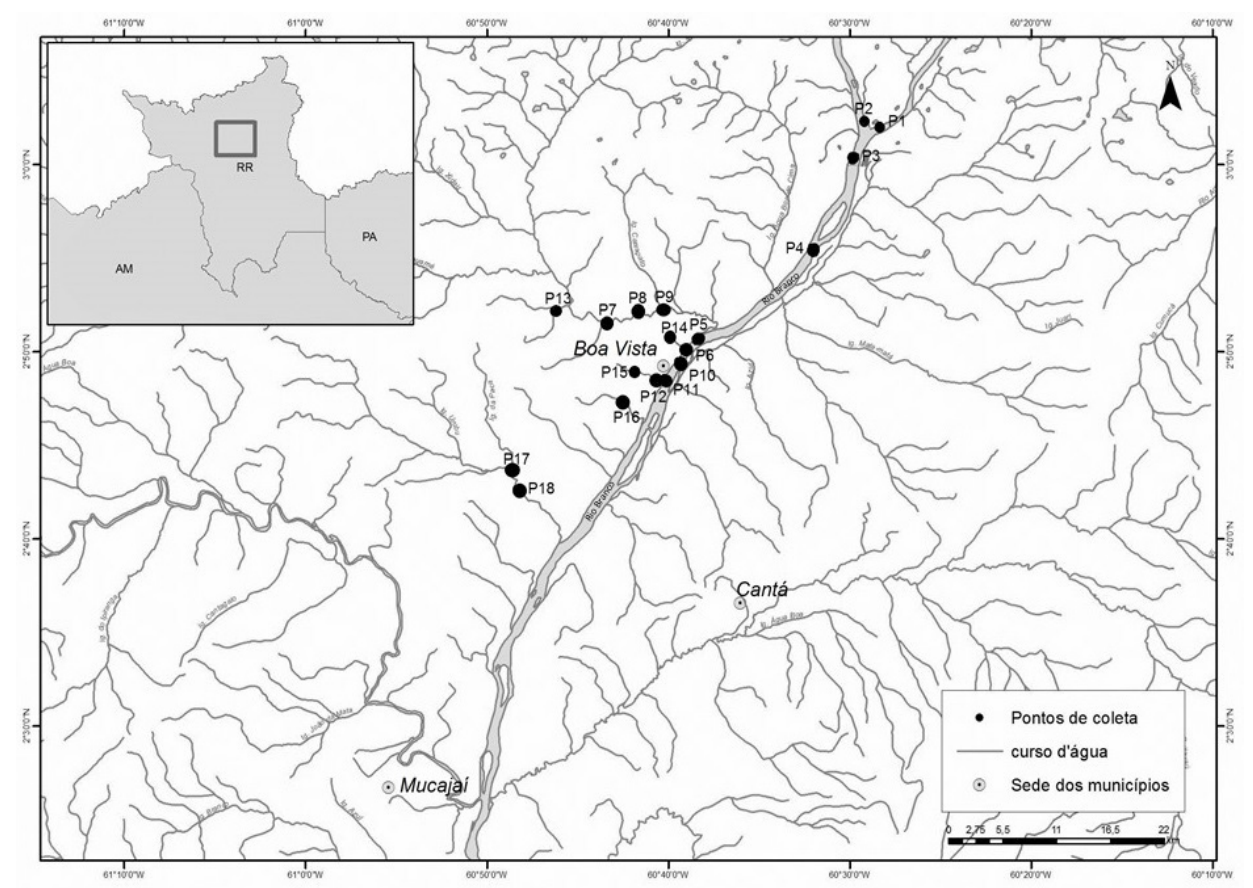

Figura 1 - Localização dos 18 pontos de coleta de água superficial no município de Boa Vista-RR.

Fonte: autora

As análises referentes a $\mathrm{pH}, \mathrm{CE}$, STD, TEMP e OD, foram realizadas por equipamentos portáteis, devidamente calibrados, no local da coleta. As demais análises físico-químicas foram realizadas no Laboratório de Águas da Universidade Federal de Roraima - UFRR, seguindo o preconizado por Standard Methods for Examination of Water and Wastewater (AMERICAN PUBLIC HEALTH ASSOCIATION, 1999).

Para a realização das análises de COL TERM, as amostras foram submetidas à técnica de tubos múltiplos para determinar o número provável de coliformes termotolerantes por $100 \mathrm{~mL}$ de água 
(NMP/100mL), conforme orientação constante em Standard Methods for Examination of Water and Wastewater (AMERICAN PUBLIC HEALTH ASSOCIATION, 1999) e Resolução 357/2005 - CONAMA (BRASIL, 2005).

O Índice de Qualidade de Água - IQA utilizado, teve como base estudo realizado por Brown et al. (1970) para "National Sanitation Foundation" dos Estados Unidos, adaptado pelo Laboratório de Águas da Universidade Federal de Roraima, para as condições naturais dos corpos hídricos locais conforme Vital (2004). Para melhor entendimento, nomeamos-lhe de IQA-RR, o qual foi determinado pelo produtório ponderado da qualidade de água correspondentes aos parâmetros: TEMP, pH, OD, $\mathrm{DBO}_{5,20}, \mathrm{COL}$ TERM, STD, $\mathrm{NO}_{3}, \mathrm{Cl}$-, ALC, CE e TURB. Os dados obtidos foram submetidos à planilha do Microsoft Office Excel produzida pelo laboratório supracitado, e os resultados obtido foram enquadrados em níveis de qualidade da água indicado na Tabela 1.

Posteriormente foi realizada análise multivariada (Análise por Componentes Principais - PCA) para determinar agrupamentos em função dos valores dos 11 parâmetros de qualidade de água observados nos 18 pontos de amostragem, nos seis meses de observação. Neste trabalho, optamos pela técnica do auto escalonamento devido à alta diferença nos valores das variáveis utilizadas. Para a escolha da proximidade entre dois agrupamentos optamos pela distância euclidiana.

Na sequência realizamos Análise Hierárquica de Clusters - HCA pelo método da distância euclidiana, com o objetivo de obter um diagrama de entrada simples em grupos formados a partir das informações obtidas dos pontos de amostragem com características comuns, de acordo com os parâmetros de qualidade de água analisados.

Tabela 1 - Níveis de qualidade da água de acordo com as faixas de variação do Índice de Qualidade da Água (IQA -RR).

\begin{tabular}{c|c}
\hline \multicolumn{2}{c}{ IQA-RR } \\
\hline Nível de Qualidade & Faixa \\
\hline Excelente & $90<\mathrm{IQA}<=100$ \\
\hline Bom & $70<\mathrm{IQA}<=90$ \\
\hline Médio & $50<\mathrm{IQA}<=70$ \\
\hline Ruim & $25<\mathrm{IQA}<=50$ \\
\hline Muito Ruim & $00<\mathrm{IQA}<=25$ \\
\hline
\end{tabular}

\section{RESULTADOS}

As médias e desvios padrão dos parâmetros de qualidade de água analisados para cada ponto de coleta encontram-se demonstrados na Tabela 2 .

Tabela 2 - Médias e desvios padrão dos parâmetros coletados para os 18 pontos de coleta.

\begin{tabular}{c|c|c|c|c|c|c}
\hline PC & $\mathbf{p H}$ & $\mathbf{C E}(\boldsymbol{\mu S} / \mathbf{c m})$ & \multicolumn{1}{c}{ STD $(\mathbf{m g} / \mathbf{L})$} & OD(mg/L) & TEMP $\left({ }^{\circ} \mathbf{C}\right)$ & DBO $_{5,20}(\mathbf{m g} / \mathbf{L})$ \\
\hline P1 & $7,18 \pm 0,20$ & $7,50 \pm 0,39$ & $8,18 \pm 1,07$ & $5,38 \pm 0,13$ & $26,92 \pm 0,62$ & $1,93 \pm 0,06$ \\
\hline P2 & $7,14 \pm 0,08$ & $7,34 \pm 1,19$ & $7,50 \pm 1,30$ & $5,18 \pm 0,13$ & $26,86 \pm 0,28$ & $1,35 \pm 0,39$ \\
\hline P3 & $6,42 \pm 0,13$ & $7,42 \pm 1,14$ & $7,64 \pm 1,57$ & $5,22 \pm 0,16$ & $26,76 \pm 0,30$ & $1,74 \pm 0,46$ \\
\hline P4 & $6,14 \pm 0,11$ & $7,72 \pm 1,00$ & $7,30 \pm 1,36$ & $5,28 \pm 0,23$ & $27,10 \pm 0,27$ & $1,93 \pm 0,06$ \\
\hline P5 & $6,31 \pm 0,13$ & $5,48 \pm 0,13$ & $5,36 \pm 0,20$ & $5,12 \pm 0,13$ & $28,24 \pm 0,82$ & $2,89 \pm 0,09$ \\
\hline P6 & $5,82 \pm 0,06$ & $60,38 \pm 7,11$ & $49,40 \pm 7,80$ & $0,64 \pm 0,32$ & $29,36 \pm 0,69$ & $35,60 \pm 3,06$ \\
\hline P7 & $6,31 \pm 0,12$ & $5,10 \pm 0,15$ & $5,14 \pm 0,23$ & $5,28 \pm 0,49$ & $28,40 \pm 0,33$ & $3,20 \pm 0,76$ \\
\hline P8 & $6,34 \pm 0,16$ & $5,12 \pm 0,14$ & $4,94 \pm 0,23$ & $5,00 \pm 0,15$ & $28,44 \pm 0,20$ & $3,70 \pm 0,43$ \\
\hline P9 & $6,10 \pm 0,22$ & $5,20 \pm 0,10$ & $4,70 \pm 0,10$ & $5,06 \pm 0,47$ & $28,54 \pm 0,47$ & $3,32 \pm 0,50$ \\
\hline P10 & $6,66 \pm 0,13$ & $10,66 \pm 0,92$ & $9,46 \pm 0,20$ & $5,30 \pm 0,12$ & $27,48 \pm 1,14$ & $2,11 \pm 0,38$ \\
\hline P11 & $6,28 \pm 0,14$ & $12,20 \pm 1,34$ & $10,24 \pm 0,27$ & $3,20 \pm 0,15$ & $27,62 \pm 1,03$ & $6,14 \pm 0,45$ \\
\hline P12 & $5,81 \pm 0,07$ & $61,68 \pm 3,93$ & $46,24 \pm 0,24$ & $3,08 \pm 0,13$ & $28,76 \pm 0,71$ & $51,77 \pm 2,38$ \\
\hline P13 & $5,69 \pm 0,09$ & $49,48 \pm 7,06$ & $42,80 \pm 8,98$ & $3,60 \pm 0,47$ & $28,62 \pm 0,48$ & $35,32 \pm 4,58$ \\
\hline P14 & $5,83 \pm 0,08$ & $59,26 \pm 6,77$ & $47,20 \pm 9,77$ & $5,34 \pm 0,21$ & $28,48 \pm 0,64$ & $32,03 \pm 2,86$ \\
\hline P15 & $5,71 \pm 0,06$ & $53,56 \pm 4,90$ & $46,68 \pm 11,39$ & $1,54 \pm 0,11$ & $28,90 \pm 0,40$ & $45,64 \pm 4,04$ \\
\hline P16 & $5,41 \pm 0,18$ & $58,70 \pm 4,60$ & $51,96 \pm 8,37$ & $1,86 \pm 0,35$ & $28,04 \pm 0,72$ & $50,34 \pm 5,11$ \\
\hline P17 & $5,80 \pm 0,07$ & $3,92 \pm 0,44$ & $3,50 \pm 0,22$ & $4,12 \pm 0,13$ & $27,48 \pm 0,34$ & $0,97 \pm 0,04$ \\
\hline P18 & $5,68 \pm 0,14$ & $3,90 \pm 0,55$ & $3,36 \pm 0,20$ & $4,12 \pm 0,08$ & $27,32 \pm 0,27$ & $0,97 \pm 0,04$ \\
\hline
\end{tabular}




\begin{tabular}{c|c|c|c|c|c}
\hline PC & $\mathbf{N O}_{3}(\mathbf{m g} / \mathbf{L})$ & ALC(mg/L) & CL-(mg/L) & TURB(NTU) & COL TERM(NMP/100mL) \\
\hline P1 & $2,50 \pm 0,71$ & $137,23 \pm 8,54$ & $13,56 \pm 2,28$ & $64,60 \pm 5,77$ & $2,50 \mathrm{E}+01 \pm 3,31$ \\
\hline P2 & $0,92 \pm 0,78$ & $127,87 \pm 17,33$ & $13,04 \pm 1,82$ & $60,80 \pm 6,55$ & $2,70 \mathrm{E}+01 \pm 5,41$ \\
\hline P3 & $0,78 \pm 0,80$ & $131,47 \pm 20,00$ & $14,37 \pm 1,46$ & $57,00 \pm 8,38$ & $2,90 \mathrm{E}+01 \pm 2,48$ \\
\hline P4 & $1,60 \pm 0,91$ & $133,34 \pm 9,86$ & $15,04 \pm 1,36$ & $58,60 \pm 5,41$ & $3,10 \mathrm{E}+01 \pm 6,19$ \\
\hline P5 & $2,01 \pm 0,86$ & $131,18 \pm 19,53$ & $15,60 \pm 1,30$ & $67,00 \pm 6,04$ & $1,16 \mathrm{E}+02 \pm 17,43$ \\
\hline P6 & $5,84 \pm 1,27$ & $123,84 \pm 12,19$ & $24,47 \pm 3,12$ & $76,40 \pm 4,41$ & $5,20 \mathrm{E}+04 \pm 13,67$ \\
\hline P7 & $2,10 \pm 0,87$ & $108,58 \pm 12,33$ & $23,78 \pm 2,18$ & $14,60 \pm 6,39$ & $2,54 \mathrm{E}+02 \pm 16,74$ \\
\hline P8 & $2,03 \pm 0,79$ & $110,45 \pm 14,58$ & $14,12 \pm 1,54$ & $14,80 \pm 3,35$ & $2,48 \mathrm{E}+02 \pm 17,04$ \\
\hline P9 & $2,24 \pm 0,97$ & $115,78 \pm 12,83$ & $14,66 \pm 2,78$ & $14,20 \pm 2,86$ & $2,60 \mathrm{E}+02 \pm 26,33$ \\
\hline P10 & $2,26 \pm 0,71$ & $137,38 \pm 12,94$ & $15,01 \pm 1,49$ & $53,80 \pm 7,19$ & $1,54 \mathrm{E}+02 \pm 38,26$ \\
\hline P11 & $2,48 \pm 0,86$ & $133,49 \pm 6,33$ & $18,85 \pm 2,07$ & $60,00 \pm 8,46$ & $1,36 \mathrm{E}+03 \pm 87,05$ \\
\hline P12 & $4,58 \pm 1,04$ & $122,11 \pm 17,22$ & $26,63 \pm 3,25$ & $77,40 \pm 8,59$ & $6,80 \mathrm{E}+04 \pm 66,00$ \\
\hline P13 & $4,01 \pm 0,95$ & $109,44 \pm 10,54$ & $23,83 \pm 1,94$ & $54,80 \pm 7,85$ & $2,00 \mathrm{E}+03 \pm 69,20$ \\
\hline P14 & $4,00 \pm 0,77$ & $126,14 \pm 8,75$ & $22,59 \pm 2,53$ & $73,60 \pm 8,89$ & $6,20 \mathrm{E}+04 \pm 96,93$ \\
\hline P15 & $4,10 \pm 1,61$ & $125,86 \pm 18,58$ & $23,22 \pm 2,53$ & $77,40 \pm 5,13$ & $7,20 \mathrm{E}+04 \pm 66,00$ \\
\hline P16 & $5,07 \pm 0,77$ & $139,68 \pm 18,05$ & $24,20 \pm 2,94$ & $82,40 \pm 6,54$ & $1,18 \mathrm{E}+05 \pm 62,84$ \\
\hline P17 & $0,60 \pm 0,84$ & $110,16 \pm 7,84$ & $14,08 \pm 1,52$ & $2,00 \pm 0,07$ & $22,00 \pm 0,44$ \\
\hline P18 & $0,52 \pm 0,28$ & $106,56 \pm 7,15$ & $14,27 \pm 1,49$ & $2,40 \pm 0,14$ & $21,00 \pm 0,55$ \\
\hline
\end{tabular}

O cálculo do IQA-RR para os 18 pontos de coleta apresentou resultados variando de zero a 88,49 (média 50,97₫37,65), indicando três níveis de qualidade da água: BOM, MÉDIO e MUITO RUIM (Tabela 3).

Tabela 3 -IQA-RR e níveis de qualidade dos 18 pontos de coleta de água em Boa Vista-RR.

\begin{tabular}{l|c|c}
\hline PONTOS DE COLETA & IQA-RR & NÍVEL \\
\hline P1 Rio Tacutu & 77,4 & BOM \\
\hline P2 Rio Uraricoera & 83,1 & BOM \\
\hline P3 Rio Branco - Igreja & 81,7 & BOM \\
\hline P4 Rio Branco - Rizicultura & 79,34 & BOM \\
\hline P5 Rio Branco - Foz Cauamé & 71,26 & BOM \\
\hline P6 Rio Branco - Foz Mirandinha & 0,0 & MUITO RUIM \\
\hline P7 Rio Cauamé - Montante igarapé Caranã & 67,36 & MÉDIO \\
\hline P8 Rio Cauamé - Ponte & 64,17 & MÉDIO \\
\hline P9 Rio Cauamé - Curupira & 65,62 & MÉDIO \\
\hline P10 Rio Branco - CAER & 77,3 & BOM \\
\hline P11 Rio Branco - Bairro Caetano Filho & 73,56 & BOM \\
\hline P12 Rio Branco - Foz igarapé Caxangá & 0,07 & MUITO RUIM \\
\hline P13 Igarapé Caranã - Foz do igarapé Caranã & 0,0 & MUITO RUIM \\
\hline P14 Igarapé Mirandinha - Drenagem & 0,08 & MUITO RUIM \\
\hline P15 Igarapé Caxangá - Elevatória CAER & 0,0 & MUITO RUIM \\
\hline P16 Igarapé Grande - Lagoa Estabilização & 0,06 & MUITO RUIM \\
\hline P17 Igarapé Água Boa - Ponte & 87,96 & BOM \\
\hline P18 Igarapé Água Boa & 88,49 & BOM \\
\hline
\end{tabular}

A análise por Componentes Principais (PCA) mostrou que a Componente Principal 1 - PC1 explicou 62,9\% da variação dos dados; a Componente Principal 2 - PC2 explicou 20,24\%, totalizando aproximadamente $83 \%$ da variação dos dados. Por isso, utilizamos os eixos 1 e 2 da PCA.

Houve formação de três grupos distintos a partir dos 18 pontos amostrados - G1, G2 e G3 (Figura 2). A PC1 separou os grupos G1 e G2 do grupo G3. As variáveis responsáveis pela separação foram $\mathrm{DBO}_{5,20}, \mathrm{CE}, \mathrm{STD}, \mathrm{NO}_{3}, \mathrm{Cl}$ - e COL TERM, nessa ordem de importância (Tabela2). A PC2 realizou a separação entre os grupos G1 e G2, sendo o pH, ALC, TURB e TEMP os parâmetros responsáveis por esta separação (Tabela 4). 
Tabela 4 - Coeficientes da estrutura da PCA para a qualidade físico-química e microbiológica da água nos 18 pontos de amostragem.

\begin{tabular}{|c|c|c|}
\hline VARIÁVEL & PC1 & PC2 \\
\hline $\mathrm{pH}$ & $-0,2355$ & 0,4062 \\
\hline $\mathrm{CE}$ & 0,3560 & 0,0125 \\
\hline STD & 0,3484 & 0,0374 \\
\hline OD & $-0,3113$ & 0,0204 \\
\hline TEMP & 0,2716 & $-0,3185$ \\
\hline DBO & 0,3583 & $-0,0228$ \\
\hline $\mathrm{NO}_{3}$ & 0,3473 & 0,0684 \\
\hline ALC & 0,0536 & 0,6709 \\
\hline $\mathrm{Cl}^{-}$ & 0,3320 & $-0,0947$ \\
\hline TURB & 0,2384 & 0,5090 \\
\hline COL TERM & 0,3267 & 0,0909 \\
\hline
\end{tabular}

Quando relacionamos o resultado da PCA (Figura 2) com os resultados do IQA-RR (Tabela 3), verificamos que o grupo G1 correspondeu aos pontos de coleta classificados como BOM pelo cálculo do IQA-RR, situados nos rios Tacutu, Uraricoera e Branco; O grupo G2 referiu-se aos pontos de coleta classificados como BOM e MÉDIO pelo cálculo do IQA-RR, situados no rio Cauamé e igarapé Água Boa; 0 grupo G3, correspondeu aos pontos classificados como MUITO RUIM pelo cálculo do IQA-RR, localizados nos igarapés Mirandinha, Caxangá, Grande e Caranã.

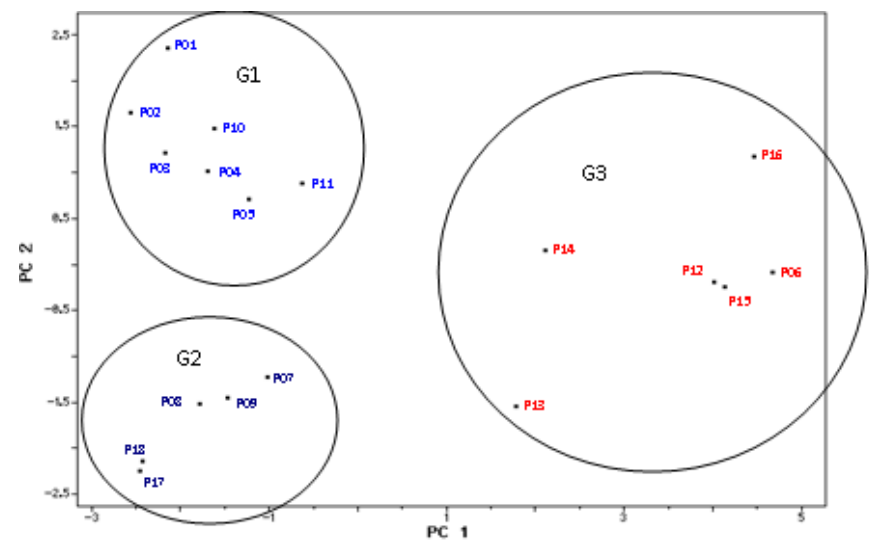

Figura 2 - Gráfico de scores (PC1/PC2) mostrando a separação dos pontos de coleta de água superficial na cidade de Boa Vista-RR.

Os resultados obtidos por meio da HCA (Figura 3) confirmaram os grupos apresentados na PCA (Figura 2), G1, G2 e G3, na linha de corte de 70\% de semelhança.

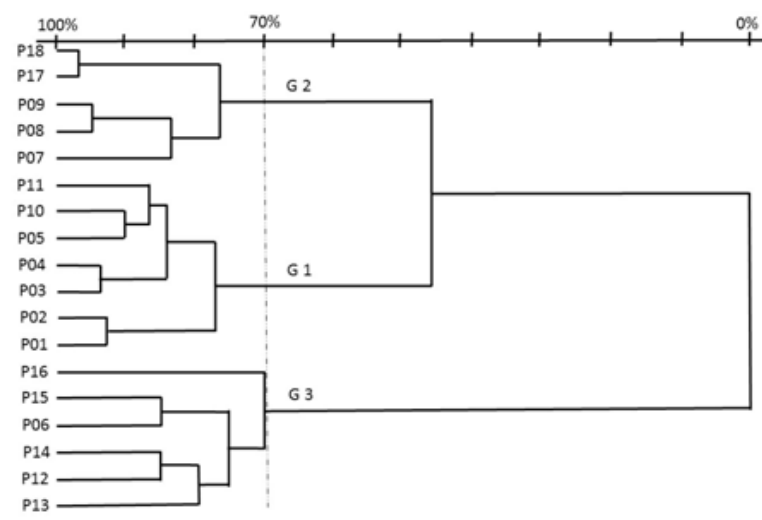

Figura 3 - Dendrograma obtido por meio da Análise Hierárquica de Cluster (HCA), apresentando agrupamento por semelhança dos 18 pontos de coleta de água superficial de Boa Vista-RR. 
As semelhanças entre os corpos hídricos de cada grupo mostrada pela HCA (Fig. 3) foram: G1- rios Tacutu, Uraricoera e Branco - com 0,78 de semelhança entre si; G2 - rio Cauamé e igarapé Água Boa com 0,78 de semelhança entre si; G3 - igarapés Mirandinha, Caxangá, Grande e Caranã - com 0,76 de semelhança entre si.

\section{DISCUSSÃO}

Nossos resultados indicaram forte relação da qualidade da água superficial com o processo de urbanização da cidade de Boa Vista. Este resultado foi concordante com investigações realizadas em diferentes regiões do Brasil (SCHUELER; SANTOS; SANTOS, 2015; MENEZES et al., 2016; CECCONELLO; CENTENO; GUEDES, 2018; VALLIM; TRAVASSOS, 2019), e em especial na Amazônia, como em cidade do estado do Pará (MELLO; ARAÚJO; SANTOS, 2018; PIMENTEL et al., 2019) e do estado do Amazonas (OLIVEIRA; OLIVEIRA; LIMA, 2018; MACHADO; ZANETI; HIGUCHI, 2019).

Nosso estudo também apontou distintos grupos que se caracterizam pelas condições da qualidade físico-química e microbiológica da água, onde o grupo com pontos fora da área urbana (G1) assinalaram relações diretas com baixa $\mathrm{DBO}_{5,20}, \mathrm{CE}, \mathrm{STD}, \mathrm{NO}_{3}, \mathrm{Cl}^{-}$e COL TERM (COMPANHIA DE TECNOLOGIA DE SANEAMENTO AMBIENTAL, 2006; MENEZES et al., 2016; DANELUZZI; GONÇALVES; FREITAS, 2018; OLIVEIRA; OLIVEIRA; LIMA, 2018). Esta é uma característica relatada por outros estudos na Amazônia (VITAL, 2004; MELLO; ARAÚJO; SANTOS, 2018; PIMENTEL et al. 2019), e por estudos internacionais em rios urbanos na China, Japão (HUANG et al., 2010; FENG et al., 2018; WAN ABDUL GHANI et al., 2018), advertindo que altos índices destas variáveis são fortes indicadores de atividades antrópicas urbanas.

Os resultados mais alarmantes referentes à poluição hídrica foram encontrados no grupo G3. A maioria dos corpos d'água pertencentes a esse grupo são pequenos afluentes do rio Branco, que cortam a zona urbana da cidade de Boa Vista e possuem como características convergentes: a ausência de mata ciliar na maior parte de seu curso, edificações próximas as margens, estreita lâmina d'água, despejo direto de efluentes domésticos e presença de lixo em seus leitos. Essas características são comuns em rios urbanos que sofrem com a poluição de suas águas (HUANG et al., 2010; DANELUZZI; GONÇALVES; FREITAS, 2018; OLIVEIRA; OLIVEIRA; LIMA, 2018; PIMENTEL et al., 2019). Assim, atenção especial deve ser dada a esse grupo de corpos hídricos, com prioridade a gestão eficiente para a melhoria da qualidade de suas águas, que está ligada diretamente ao planejamento urbano e gestão social.

Todo esse processo de degradação ocorrido nos corpos hídricos constantes no grupo G3 acabou por refletir na diminuição gradual da qualidade da água do rio Branco (grupo G1), conforme sua aproximação com a zona urbana da capital. Mesmo possuindo um volume d'água superior aos seus afluentes urbanos e tendo o efeito de autodepuração amenizando a carga poluidora recebida (SCHUELER; SANTOS; SANTOS, 2015; CUNHA; FERREIRA, 2019), quando comparados com Vital (2004), nossos resultados mostraram notória redução da qualidade das águas do rio Branco com o passar do tempo, especialmente em suas porções próximas e dentro da área urbana de Boa Vista, o que também é corroborado por Mello, Araújo e Santos (2018), em estudos acerca dos processos de urbanização de cidade de Marabá-PA e os reflexos no rio Tocantins.

Quando analisamos o grupo G2, percebemos que mesmo com IQA-RR mais elevado, os seus corpos hídricos começaram a sofrer processos de degradação. Destacamos aqui a distinção na qualidade de água verificada entre o rio Cauamé, que apresentou menor qualidade de água quando comparado ao igarapé Água Boa. Tal diferenciação pode ser explicada pela localização dos pontos do rio Cauamé situados em ambientes utilizados como balneários públicos, com intensa atividade antrópica. Interferência na qualidade da água provocada por balneários também foi verificada por Amaral e Silva (2017), em balneário em Rondônia e por Alves et al. (2018), em cursos d’água no Pará.

Analisando conjuntamente os grupos G1, G2 e G3 nosso estudo mostrou a distribuição espacial dos corpos hídricos em relação ao seu nível de qualidade, apontando que ela estava diretamente ligada ao processo de urbanização, ficando claro a ausência do cuidado ambiental e social adequado influenciando negativamente a qualidade da água superficial urbana da cidade de Boa Vista.

\section{CONCLUSÕES}

As avaliações realizadas classificaram os pontos analizados dentro de três grupos distintos: G1 com bom nível de qualidade, situado fora do ambiente urbano; G2 - com nível médio de qualidade, próximo ao ambiente urbano e com presença de balneários públicos e G3 - com nível de qualidade 
muito ruim, inserido no ambiente urbano, evidenciando a influência negativa da urbanização na qualidade de água superficial da cidade.

Os parâmetros que mais influenciaram na qualidade da água dos pontos amostrados foram: $\mathrm{DBO}_{5,20}, \mathrm{CE}, \mathrm{STD}, \mathrm{NO}_{3}, \mathrm{Cl}^{-}$e COL TERM, estando, os altos índices dessas variáveis, relacionados diretamente com processos ligados a urbanização.

Deve ser dada atenção especial ao controle de poluição e tratamento de efluentes despejados nos corpos hídricos situados no grupo G3, os quais apresentaram problemas mais alarmantes na degradação de sua qualidade.

Programa de monitoramento permanente deve ser adotado, considerando as estações do ano, para determinação da qualidade da água superficial de Boa Vista, a fim proporcionar informações robustas que auxiliem na formulação de políticas públicas para mitigação dos efeitos negativos da urbanização sobre os recursos hídricos da Capital.

\section{REFERÊNCIAS}

ALVARES, C. A. et al. Köppen's climate classification map for Brazil. Meteorologische Zeitschrift, Berlin, v. 22, p. 711-728, 2014.

ALVES, J. D. N. et al. Avaliação da qualidade da água em cursos d' água no município de Capitão Poço - PA. Nucleus, Ituverava, v. 15, n. 1, p. 269-277, 2018.

AMARAL, C. T.; SILVA, F. S. A. Qualidade da água em um igarapé balneário na Amazônia: estudo de caso em Porto Velho. InterEspaço, Grajaú, v. 3, n. 8, p. 251-267, 2017.

AMERICAN PUBLIC HEALTH ASSOCIATION - APHA. Standard methods for examination of water and wastewater. 19th ed. Baltimore: United Book Press, 1999.

ARAÚJO JUNIOR, A. C. R.; TAVARES JUNIOR, S. S. Expansão urbana e fatores de risco à inundação em Boa Vista RR. Revista Raega, Curitiba, v. 44, p. 139-153, 2018.

BARBOSA, R. I.; CAMPOS, C. Detection and geographical distribution of clearing areas in the savannas ('lavrado') of Roraima using Google Earth web tool. Journal of Geography and Regional Planning, Nairobi, v. 4, p. 122136, 2011.

BECKER, B. K. A especificidade do urbano na Amazônia: desafios para políticas públicas consequentes. Estudo elaborado para a Secretaria de Coordenação dos Assuntos da Amazônia Legal. Rio de Janeiro: Ministério do Meio Ambiente, 1998.

BECKER, B. K. Amazônia: mudanças estruturais e urbanização. In: GONÇALVES, M. F. (Ed.). Regiões e cidades, cidades nas regiões. São Paulo: ANPUR/UNESP, 2003. v. 1.

BRASIL. Resolução ${ }^{\circ}$ 357, de 17 de março de 2005. Dispõe sobre a classificação dos corpos de água e diretrizes ambientais para o seu enquadramento, bem como estabelece as condições e padrões de lançamento de efluentes, e dá outras providências. Diário Oficial [da] República Federativa do Brasil, Poder Executivo, Brasília, DF, 2005.

BROWN, R. M. et al. A water quality index: do we dare? Water \& Sewage Works, Chicago, v. 117, n. 10, 1970.

CARVALHO, T. M.; MORAIS, R. P. Aspectos hidrogeomorfológicos do sistema fluvial do baixo rio Uraricoera e Alto rio Branco como subsídio à gestão de terras. Geografias, Belo Horizonte, v. 10, n. 2, p. 118-135, 2014.

CECCONELLO, S. T.; CENTENO, L. N.; GUEDES, H. A. S. Índice de qualidade de água modificado a partir da análise estatística multivariada: estudo de caso do Arroio Pelotas, RS. Engenharia Sanitaria e Ambiental, Rio de Janeiro, v. 23, n. 5, 2018.

COMPANHIA DE TECNOLOGIA DE SANEAMENTO AMBIENTAL - CETESB. Índice de Qualidade da Água: IQA. Rio de Janeiro, 2006. Disponível em: <http://www.cetesb.gov.br/agua>. Acesso em: 12 jun. 2017.

CUNHA, C. L. N.; FERREIRA, A. P. Análise crítica por comparação entre modelos de qualidade de água aplicados em rios poluídos: contribuições à saúde, água e saneamento. Engenharia Sanitaria e Ambiental, Rio de Janeiro, v. 10, p. 1-8, 2019.

DANELUZZI, N. S.; GONÇALVES, V. D.; FREITAS, R. P. Avaliação da qualidade da água de uma bacia hidrográfica urbana: estudo de caso bacia do rio Pinhalzinho II, Umuarama - PR. Revista de Ciências Ambientais, Canoas, v. 14, n. 3, p. 678-698, 2018.

FALCÃO, M. T. et al. Impactos ambientais no igarapé Wai grande em Boa Vista - Roraima decorrentes da influência do aterro sanitário. Revista Geonorte, Manaus, v. 3, n. 6, p. 199-207, 2012. 
FENG, H. et al. Threshold analysis of urbanization with the constraint of "Three Red Lines" on water resources management: a case study of Zhangye City. Ziran Ziyuan Xuebao, Beijing, v. 33, n. 2, p. 287-301, 2018.

HUANG, F. et al. Spatial variation and source apportionment of water pollution in Qiantang River (China) using statistical techniques. Water Research, Oxford, v. 44, n. 5, p. 1562-1572, 2010.

INSTITUTO BRASILEIRO DE GEOGRAFIA E ESTATÍSTICA - IBGE. Censo demográfico 2010. Rio de Janeiro, 2010. Disponível em: <http://www.ibge.gov.br>. Acesso em: 12 jun. 2017.

LUCKMANN, D. História e geografia do município de Boa Vista. Boa Vista: Grafima, 1989. (Série Roraima Através dos Municípios).

MACHADO, A. L. S.; ZANETI, I. C. B. B.; HIGUCHI, M. I. G. A degradação dos cursos hídricos urbanos, uma abordagem sobre gestão e educação ambiental. Revista Ibero-Americana de Estudos em Educação, Araraquara, v. 14, n. 3, p. 1124-1138, 2019.

MELLO, A. H.; ARAÚJO, E. V. N.; SANTOS, J. S. O processo de urbanização de Marabá e os reflexos sobre o Rio Tocantins e suas relações. Agenda Social, Campos dos Goytacazes, v. 12, n. 1, p. 191-201, 2018.

MENEZES, J. P. C. et al. Relação entre padrões de uso e ocupação do solo e qualidade da água em uma bacia hidrográfica urbana. Engenharia Sanitaria e Ambiental, Rio de Janeiro, v. 21, n. 3, p. 519-534, 2016.

OLIVEIRA, L. F.; OLIVEIRA, B. O. S.; LIMA, L. B. Avaliação da qualidade da água de três córregos na área urbana de Humaitá-AM. Revista de Ciências Ambientais, Canoas, v. 12, n. 3, p. 25-33, 2018.

PIMENTEL, D. R. et al. Avaliação da qualidade da água do Igarapé do Urumari, Santarém, Pará. Revista Saúde e Meio Ambiente, Três Lagoas, v. 8, n. 1, p. 153-161, 2019.

SCHUELER, A.; SANTOS, F. V. F.; SANTOS, V. P. Contaminação dos rios urbanos. Cadernos de Pós-graduação em Arquitetura e Urbanismo, São Paulo, v. 15, n. 1, p. 109-126, 2015.

SILVA, D. et al. Análise dos ciclos de precipitação na região de Boa Vista - RR nos anos de 1910 a 2014. Revista Geográfica Acadêmica, Boa Vista, v. 9, p. 35-49, 2015.

TOSCANO, G. G.; SILVA, T. C. S. Uma síntese analítica sobre legislação de proteção das águas subterrâneas. Gaia Scientia, João Pessoa, v. 6, p. 1-20, 2012.

TOZI, S. C.; MASCARENHAS, A. L.; PÓLEN, R. R. Água, conflitos e política ambiental na Amazônia legal brasileira. Revista Nera, Presidente Prudente, v. 21, n. 42, p. 228-255, 2018.

TUNDISI, J. G. Recursos hídricos no Brasil: problemas, desafios e estratégias para o futuro. Rio de Janeiro: Academia Brasileira de Ciências, 2014.

VALLIM, E. M.; TRAVASSOS, L. R. F. C. Impasses sobre a urbanização e a produção de água no sistema produtor alto Tietê: estudos sobre a evolução da mancha urbana e impactos ambientais no município de Suzano-SP. Revista Geografia em Atos, Presidente Prudente, v. 1, n. 9, p. 5-22, 2019.

VITAL, M. J. S. Boa Vista-RR: o monitoramento dos recursos hídricos e a necessidade de indicadores ambientais. 2004. Tese - Centro de Ciências Biológicas. Universidade Federal de Roraima, Boa Vista, 2004.

WAN ABDUL GHANI, W. M. H. et al. Performance of biotic indices in comparison to chemical-based Water Quality Index (WQI) in evaluating the water quality of urban river. Environmental Monitoring and Assessment, Dordrecht, v. 190, n. 5, p. 297, 2018.

\section{Contribuições dos autores}

Andréa Cristina Sant'Ana: Escrita, coleta e análise de dados.

Marcos José Salgado Vital: Orientação, revisão e acompanhamento da pesquisa.

Henrique Eduardo Bezerra da Silva: Coorientação e acompanhamento da pesquisa. 